\title{
Modulation of postural tremors at the wrist by supramaximal electrical median nerve shocks in essential tremor, Parkinson's disease and normal subjects mimicking tremor
}

\author{
T C Britton, P D Thompson, B L Day, J C Rothwell, L J Findley, C D Marsden
}

\begin{abstract}
The response of postural wrist tremors to supramaximal median nerve stimulation was examined in patients with hereditary essential tremor $(n=10)$ and Parkinson's disease $(n=9)$, and in normal subjects mimicking wrist tremor $(n=8)$. The average frequency of on-going tremor was the same in all three groups. Supramaximal peripheral nerve shocks inhibited and then synchronised the rhythmic electromyographic (EMG) activity of all types of tremor. The duration of inhibition ranged from 90 to $210 \mathrm{~ms}$, varying inversely with the frequency of on-going tremor. There was no significant difference in mean duration of inhibition or in the timing of the first peak after stimulation on the average rectified EMG records between the three groups. The degree to which supramaximal peripheral nerve shocks could modulate the timing of rhythmic EMG bursts in the forearm flexor muscles was also quantified by deriving a resetting index. No significant difference in mean resetting index of the three groups was found. These results suggest that such studies cannot be used to differentiate between the common causes of postural wrist tremors.
\end{abstract}

$(\Im$ Neurol Neurosurg Psychiatry 1993;56:1085-1089)

Examination of the period of inhibition of rhythmic EMG following supramaximal electrical peripheral nerve stimulation has been suggested as a means of distinguishing low frequency essential tremor from Parkinsonian postural tremor. ${ }^{1}$ Supramaximal electrical peripheral nerve stimulation inhibits rhythmic EMG activity in Parkinsonian rest tremor for a period of around $200 \mathrm{~ms}$, but in essential tremor rhythmic EMG activity is only inhibited for a period of about $100 \mathrm{~ms} .{ }^{1}$ The technique would be of major clinical value if it could reliably distinguish between essential tremor and Parkinsonian tremor.

The finding of differences in the response of essential and Parkinsonian postural tremors to similar stimuli would also provide evidence of differences in the physiological mechanisms underlying each tremor. Until now, no behavioural differences between essential tremor and Parkinsonian postural tremor have been reported. Both types of tremor have similar frequencies ${ }^{2}$ which are not affected by inertial loading of the limb. ${ }^{3}$
In a previous study, ${ }^{4}$ we found that both types of tremor were similarly modulated by brief mechanical perturbations when due allowance was made for ongoing tremor amplitude and perturbation size.

We set out to investigate the effect of supramaximal peripheral nerve shocks on postural tremors. To make the study as practical as possible, we ensured that all wrist tremors were studied under identical conditions. In addition to measuring the period of inhibition of rhythmic EMG produced by supramaximal median nerve stimulation, we also measured the degree to which the timing of subsequent EMG bursts were influenced by calculating a "resetting index".

\section{Methods}

\section{Patients and subjects}

Ten patients (mean age 56 years; range 30 to 76 years) with hereditary essential tremor (postural tremor most marked or only present in the upper limbs, with a positive family history of a similar disorder in at least 2 consecutive generations) and nine patients (mean age 57 years; range 34 to 73 years) with Parkinson's disease (defined as an akinetic rigid syndrome of asymmetric onset and responsive to levodopa) and tremor were studied with local ethical committee approval. All of these patients had taken part in our previous study. ${ }^{4}$ Patients with essential tremor had tremor in the outstretched arms but no tremor at rest. The four patients with essential tremor who were taking beta-blockers were asked to stop their drugs for three days before the study. All the patients with Parkinson's disease had tremor at rest and a slightly faster tremor of the outstretched arms. Anti-Parkinsonian medications were not stopped.

Eight hospital and laboratory personnel (mean age 35 years; range 31 to 39 years) without tremor or a family history of tremor were asked to mimic tremor by rapidly flexing and extending their wrists. The frequency of movement chosen by each subject ranged from $5 \cdot 5$ to $6 \cdot 5 \mathrm{~Hz}$.

\section{Recording}

Patients and subjects were seated comfortably and their semipronated arms placed in a manipulandum of low inertia coupled to a torque motor (Printed Motors type G12M4$H)$. The forearm and hand were secured to restrict movement to the wrist in a horizontal plane. The position of the manipulandum was 
displayed as a vertical bar on the lower half of an oscilloscope screen placed in front of the patient or subject: instructions were given to keep this vertical bar under a second stationary vertical bar in the centre of the screen. The apparatus was arranged so that when the two vertical bars were in line the wrist was in approximately 15 degrees of flexion, a position which was generally found to be optimal for bringing out wrist tremor in patients. Specific instructions were given to both patients and normal subjects that they should not react to the peripheral nerve stimuli.

The median nerve was stimulated at the elbow using $0.5 \mathrm{~ms}$ square-wave electrical pulses of sufficient intensity to produce a maximal EMG response in the forearm flexor muscles. Fifty stimuli were given randomly at 5 to 8 second intervals, while a constant background torque of $0.38 \mathrm{Nm}$ was maintained against the forearm flexor muscles to bring out the tremor.

Wrist position and velocity, derived by electrical differentiation of the position trace, were recorded 2 seconds before and 2 seconds after the peripheral nerve shock. EMG recordings were taken from flexor carpi radialis using $\mathrm{Ag} / \mathrm{AgCl}$ electrodes taped 3 to 4 centimetres apart over the muscle and then amplified and processed (Digitimer D160; bandpass filtering between $80 \mathrm{~Hz}$ and $2 \cdot 5 \mathrm{kHz}$ ). EMG signals were subsequently full-wave rectified and smoothed (time constant $10 \mathrm{~ms}$ ). All channels were collected by a CED $1401 \mathrm{D} / \mathrm{A}$ converter at a sampling rate of $150 \mathrm{~Hz}$ per channel before being stored on floppy disc by an IBM compatible personal computer for later analysis.

\section{Analysis}

A resetting index was derived by a method similar to that of Lee and Stein. ${ }^{5}$ Each trial was reviewed, the flexor EMG signal being subjected to multiple 3-point digital smoothing before being displayed on the computer screen. When clearly defined EMG bursts could be identified before and after the peripheral nerve shock, the timing of the peaks of the 5 bursts before and 5 bursts after the shock were measured by visual inspection using a cursor.

From the timing of the peaks of the 5 bursts before the peripheral nerve shock, the average period between bursts was calculated for each trial. The timing of the peripheral nerve shock relative to the timing of the last EMG burst before the peripheral nerve shock was then expressed as a proportion of the average cycle length. Predicted timings for the subsequent 5 EMG bursts (had there not been a peripheral nerve shock) were calculated based on the timing of the last EMG burst before the peripheral nerve shock and on the average cycle length, from which the difference between the actual timings could be calculated. A graph was then plotted of timing of the peripheral nerve shock in the on-going tremor cycle against the actual minus expected timing of the subsequent 5 EMG bursts. For each EMG burst after the peripheral nerve shock, a linear regression line was derived. The slope of the lines gives an indication of the resetting that has occurred; a slope of 0 implies no resetting, whereas a slope of 1 implies complete resetting. ${ }^{5}$ The resetting index (RI) was calculated by taking the average slope of the regression lines calculated for the first five EMG bursts following the peripheral nerve shock.

The average cycle length before the peripheral nerve shock and the average length of each of the first four cycles following the shock were calculated for each individual. The ongoing tremor frequency ranged from $4 \cdot 1$ to $7 \cdot 6 \mathrm{~Hz}$ (mean $5 \cdot 6 \mathrm{~Hz}$ ) for the essential tremor group and $4 \cdot 3$ to $6 \cdot 2 \mathrm{~Hz}$ (mean $5 \cdot 2 \mathrm{~Hz}$ ) for the Parkinson's disease group. The frequency of tremor mimicked by normal subjects ranged from 5.5 to $6 \cdot 5 \mathrm{~Hz}$ (mean $6 \mathrm{~Hz}$ ). Mean tremor amplitude was calculated by using a computer to find the positions of maximum wrist flexion and extension in the last complete cycle before each median nerve stimulus. Mean amplitude of tremor in the essential tremor group varied between 1 degree and 11 degrees (mean 3 degrees), in the Parkinson's disease group between 1 degree and 46 degrees (mean 8 degrees) and in normal subjects mimicking tremor between 2 degrees and 20 degrees (mean 10 degrees).

Statistical comparison of group data was performed with one-way analysis of variance. Correlation was performed using linear regression analysis. Regression lines were compared by analysis of covariance. Significance was judged at a $5 \%$ level.

\section{Average rectified EMG traces}

The rectified forearm flexor EMG of each trial was averaged by computer. Because peripheral nerve shocks were given at random times within the tremor cycle, this has the effect of "averaging out" the EMG bursts preceding the peripheral nerve shocks and the average rectified EMG trace in the period before the peripheral nerve shocks is therefore approximately level. The variable amount of noise in the average rectified EMG traces before the median nerve shock (see fig 1) is attributable to different patterns of rhythmic EMG activity associated with tremor in different individuals. Some individuals had large amplitude, discrete EMG bursts separated by clear silence, whereas others had smaller, more diffuse EMG bursts with some background EMG activity occurring between bursts. Large, discrete EMG patterns were associated with more noise in the average rectified EMG traces, while smaller and less discrete EMG patterns were associated with less noise.

If peripheral nerve shocks have no effect on the tremor, there would be a level trace in the period after the peripheral nerve shock as well. However, if the EMG activity in the forearm muscle is modulated in a consistent manner (for example, phase reset) by the peripheral nerve shock, then the average rectified EMG trace in the period after the peripheral nerve shock will show such modu- 


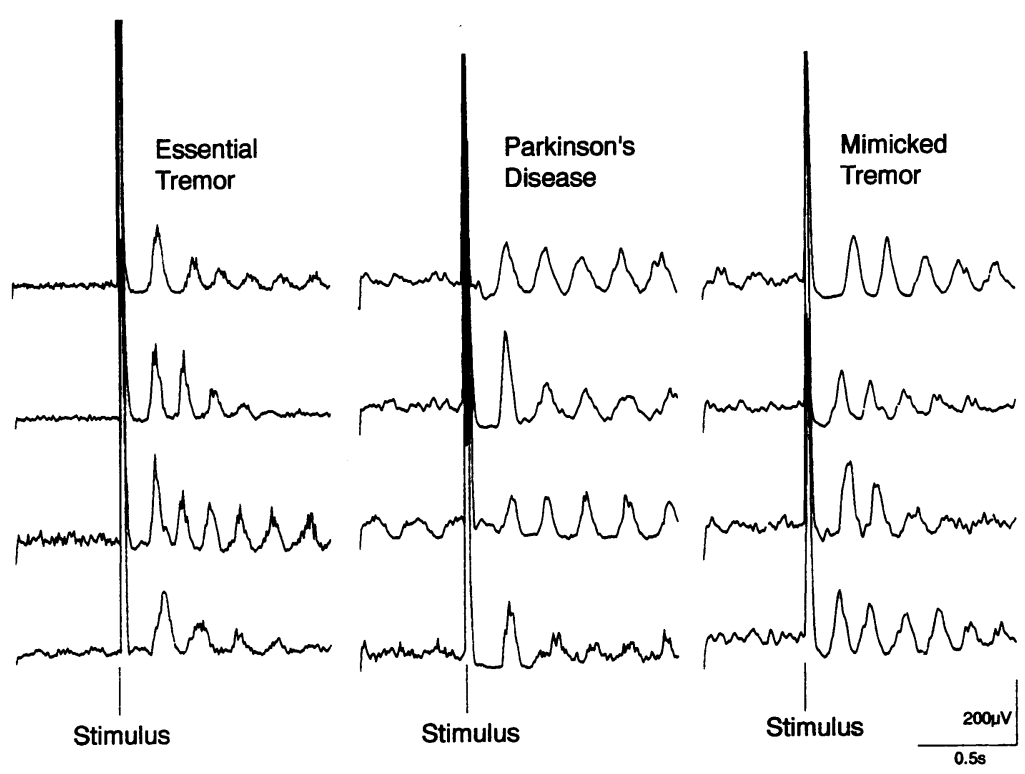

Figure 1 Effect of supramaximal electrical stimulation of the median nerve at the elbow on the postural wrist tremor of four representative patients with essential tremor (left panel), four representative patients with Parkinsonian tremor (centre panel) and four representative normal subjects mimicking tremor (right panel). Each trace is the average of 50 epochs of rectified forearm flexor EMG recorded for 0.5 seconds before and 1 second after the delivery of a supramaximal electrical stimulus to the median nerve at the elbow. The direct EMG response is concealed within the stimulus artefact and is then followed by a period during which EMG activity is suppressed, before phasic EMG modulation appears time-locked to the nerve shock. This phasic EMG modulation has a periodicity which is similar to that of the preceding tremor in each case and reflects the degree to which the timing of rhythmic EMG bursts in the forearm flexor muscles has been influenced by the nerve shock. There is no consistent difference in the behaviour of tremors of different origins to nerve stimulation. To bring out the wrist tremor in patients with essential tremor, a background torque of $0.38 \mathrm{Nm}$ was applied in such a direction as to extend the wrist: $a$ similar torque was applied when studying the effect of median nerve stimulation on the wrist tremors of patients with Parkinson's disease and of normal subjects imitating tremor.

lation. Modulation of the average rectified EMG trace usually does not last more than a few "cycles" (see figure 1). Although there are several explanations for the reduction in amplitude of the modulations with time following the stimulus, ${ }^{4}$ the effect can largely be accounted for by the inherent variability of tremors, particularly rapid small amplitude essential tremors.

The duration of inhibition of rhythmic EMG produced by median nerve stimulation was measured by visual inspection of the average rectified EMG traces and was taken as the period between the stimulus and the onset of the first peak in the average rectified EMG trace. As such, this period also includes the direct EMG response produced by the median nerve stimulus. The onset of the first peak was usually well defined, but where it was not clear, the onset of the first peak was determined by extrapolation of the slope of the first peak (at $50 \%$ peak size) back to a line representing the average height (above the baseline) of the rectified EMG trace during the preceding period of EMG suppression. The latency of the first peak in the average rectified EMG trace following median nerve stimulation was measured by visual inspection using a cursor.

\section{Results}

1) Appearance of average rectified EMG traces Supramaximal median nerve stimulation at the elbow inhibited and then synchronised the EMG bursts in the forearm flexor muscles in all the individuals studied, irrespective of tremor frequency or amplitude (fig 1). There was no difference between essential tremor, Parkinson's disease and mimicked tremor groups in their susceptibility to modulation as judged by the appearance of the average rectified EMG traces.

\section{2) Duration of inhibition of rhythmic EMG} activity

The duration of inhibition of rhythmic EMG activity following supramaximal median nerve stimulation varied between $90 \mathrm{~ms}$ and $210 \mathrm{~ms}$. The mean duration of inhibition was similar for the essential tremor (145ms), Parkinson's disease $(141 \mathrm{~ms})$ and mimicked tremor (143ms) groups (ANOVA, $\mathrm{F}=0.07, \mathrm{df}=2$, NS). Within each group, the duration of inhibition of rhythmic EMG was found to correlate significantly with the period of the on-going tremor (fig 2; for essential tremor group, linear correlation coefficient $($ Rval $)=$ $0.81, \mathrm{p}<0.005$; for Parkinson's disease group, Rval $=0.69, \mathrm{p}<0.05$; for mimicked tremor group, Rval $=0.81, \mathrm{p}<0.005)$. There was no significant difference in the slopes (essential tremor vs Parkinsonian tremor, $\mathbf{F}=$ $0 \cdot 45$, df $=1,15$, NS; essential tremor vs mimicked tremor, $\mathrm{F}=0.004, \mathrm{df}=1,14, \mathrm{NS}$ ) or elevations (essential tremor vs Parkinsonian tremor, $\mathrm{F}=2.07, \mathrm{df}=1,16, \mathrm{NS}$; essential tremor vs mimicked tremor, $\mathrm{F}=1 \cdot 28, \mathrm{df}=$ $1,15, \mathrm{NS}$ ) of the regression lines for each group.

\section{3) Resumption of EMG activity}

The latency of the first peak in the average rectified EMG varied between $150 \mathrm{~ms}$ and $270 \mathrm{~ms}$. The mean latency was similar for essential tremor (198ms), Parkinson's disease $(193 \mathrm{~ms})$ and mimicked tremor (195ms) groups (ANOVA, $\mathrm{F}=0.06, \mathrm{df}=2, \mathrm{NS}$ ). For individuals within each group the latency of the first peak in the average rectified EMG trace was found to correlate significantly with the period of on = going tremor (fig 2; for essential tremor group, linear correlation coefficient $(\mathrm{Rval})=0.84, \mathrm{p}<0.005$; for Parkinson's disease group, Rval $=0.80, \mathrm{p}<$ 0.02 ; for mimicked tremor group, Rval = $0.70, p<0.05)$. There was no significant difference in the slopes (essential tremor vs Parkinsonian tremor, $\mathrm{F}=0.24, \mathrm{df}=1,15$, NS; essential tremor vs mimicked tremor, $F$ $=0.38, \mathrm{df}=1,14, \mathrm{NS}$ ) or elevations (essential tremor vs Parkinsonian tremor, $F=2 \cdot 88$, $\mathrm{df}=1,16, \mathrm{NS}$; essential tremor vs mimicked tremor, $\mathrm{F}=1 \cdot 10, \mathrm{df}=1,15, \mathrm{NS}$ ) of the regression lines for each group.

\section{4) Resetting index}

The calculated resetting index ranged from 0.66 to 0.99 for patients with essential tremor, from 0.6 to 0.99 for patients with Parkinson's disease and from 0.88 to 0.99 for normal subjects mimicking tremor. There was no significant difference between the mean resetting indexes for the essential 


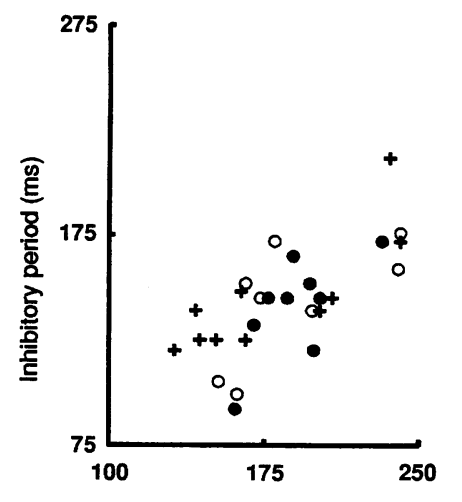

Tremor period before stimulus (ms)

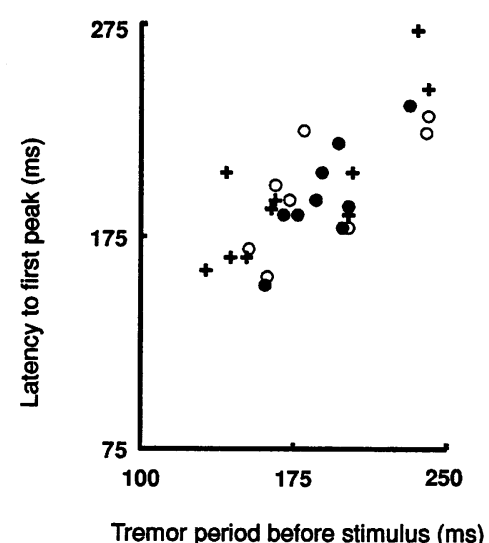

Figure 2 Left panel. Graph of the average tremor period preceding median nerve stimulation against the duration of inhibition of rhythmic EMG for patients with essential tremor (+), patients with Parkinson's disease (O) and normal subjects imitating tremor (O). Right panel. Graph of average tremor period against the latency of the first peak in the average rectified EMG trace following median nerve stimulation. The duration of inhibition of rhythmic EMG and the latency of the first peak in the average rectified EMG traces correlated significantly with the preceding tremor period for each group (see text). ence in tremor frequencies in the two patient groups, taken in conjunction with our finding that the duration of inhibition depends on the frequency of ongoing tremor might contribute to the differences in the duration of inhibition of rhythmic EMG between the two groups observed by Bathien et al. ${ }^{1}$ Second, these authors concentrated on the resting tremor of Parkinson's disease and the (presumably) postural tremor of essential tremor; in the present study both groups were examined under identical conditions (while maintaining a posture). These methodological differences may be crucially important since isometric voluntary contraction is known to reduce the duration of EMG suppression. ${ }^{1}$ Lastly, Bathien et al studied rhythmic EMG bursts in the forearm extensor muscles and stimulated the radial nerve, whereas we studied forearm flexor muscle EMG and stimulated the median nerve, but it seems unlikely that this could provide a full explanation for the difference in results.

tremor (mean resetting index $=0.92$ ), Parkinson's disease $(0.89)$ or mimicked tremor $(0.95)$ groups (ANOVA, $F=0.76$, df $=2$, NS). The resetting index did not correlate with either tremor amplitude or tremor frequency in any group.

\section{Discussion}

The main conclusion of this study is that the forearm EMG activity associated with the postural wrist tremors of essential tremor and Parkinson's disease and even the forearm EMG activity associated with wrist tremors mimicked by normal subjects is similarly modulated by supramaximal median nerve stimulation at the elbow. No difference in the behaviour of essential and Parkinsonian tremors to supramaximal nerve shocks was observed. We could not therefore confirm the previous suggestion that the technique might be useful in distinguishing low frequency essential tremor from postural Parkinsonian tremors. $^{1}$

\section{Previous studies}

This study has confirmed that postural tremors of the arm, whether essential ${ }^{1}$ or Parkinsonian tremors ${ }^{167}$ can be modulated by supramaximal nerve stimulation. However, whereas Bathien et al ${ }^{1}$ found that the duration of inhibition of rhythmic EMG following the nerve shock depended on the type of tremor, we found that the duration of inhibition of rhythmic EMG varied inversely with the frequency of on-going tremor. There are several possible explanations for this. First, we studied tremors of similar frequencies (mean essential tremor frequency $=5.6 \mathrm{~Hz}$; mean Parkinsonian tremor frequency $=$ $5 \cdot 2 \mathrm{~Hz}$ ) whereas the mean frequencies in the two groups of Bathien and colleagues' study were significantly different (mean essential tremor frequency $=9 \mathrm{~Hz}$; mean Parkinsonian tremor frequency $=5.4 \mathrm{~Hz}$; Student's unpaired $t$ test, $\mathrm{t}=4 \cdot 8, \mathrm{p}<0.0001)$. This differ-

\section{Physiological mechanisms}

What physiological mechanisms underlie the inhibition of rhythmic EMG produced by supramaximal peripheral nerve shocks? Hufschmidt $^{6}$ and later McLellan ${ }^{8}$ found that the duration of inhibition of rhythmic EMG activity produced by peripheral electrical stimuli in patients with Parkinson's disease could last up to $200 \mathrm{~ms}$ with a mean of $134 \mathrm{~ms} .{ }^{8}$ Similar values were found in our study. They suggested that the inhibition was produced by a mechanism similar to that responsible for the "silent period" in voluntary muscle activity that follows peripheral nerve stimulation as described by Merton. ${ }^{9}$ Since this "silent period" usually has a duration of only $100 \mathrm{~ms}$, Hufschmidt ${ }^{6}$ and $\mathrm{McLellan}^{8}$ proposed that the longer periods of inhibition seen in their patients might be due to an abnormality of autogenous motor neuron inhibition in Parkinson's disease. The present study questions this explanation since periods of silence lasting longer than $100 \mathrm{~ms}$ following supramaximal peripheral nerve stimulation were not limited to patients with Parkinson's disease, but were also seen in patients with essential tremor and in normal subjects mimicking tremor. Periods of inhibition longer than the "silent period" therefore were not specific to a disease, but appeared to be related to the presence of (involuntary or voluntary) oscillatory EMG activity. This finding would favour the existence of an inhibitory mechanism common to all groups rather than any disease-specific changes in autogenous inhibition. The observation that the duration of inhibition (90ms to $210 \mathrm{~ms}$ ) was variable and related to the frequency of on-going tremor makes it unlikely that the mechanism is the same as that responsible for the "silent period" which has a relatively fixed duration of around $100 \mathrm{~ms} .{ }^{9}$

Why should the period of inhibition of rhythmic EMG produced by a supramaximal peripheral nerve shock be related to the ongoing tremor frequency? The simplest expla- 
nation is that the oscillator responsible for the tremor has been "reset" to some fixed point in its cycle by the peripheral nerve shocks, irrespective of the time in the on-going cycle when the shock is given. This results in suppression of phasic drive to the muscles for a period of just less than the cycle length of the on-going tremor; following this period phasic EMG activity then resumes, time-locked to the stimulus. Analogous behaviour is exhibited by cardiac pacemaker tissue depolarised by external electrical shocks. Irrespective of when external electrical shocks are given, the cardiac pacemaker is reset, so that activity resumes a) time-locked to the shocks and b) after a period which is dependent upon the length of the cardiac cycle. Whether tremors are truly "reset" like cardiac pacemaker tissue remains uncertain. Nevertheless, the findings of our study do favour a close interaction between supramaximal peripheral nerve shocks and the oscillators responsible for essential and Parkinsonian postural tremors as well as any oscillatory mechanisms utilised by normal subjects mimicking tremor.

Not all tremors, however, were equally susceptible to modulation by peripheral nerve stimulation as judged by the individual resetting indexes which ranged from 0.6 to 0.99 . The cause of this variability in susceptibility is uncertain, but at least two factors are likely to be of importance: the size of the stimulus ${ }^{10}$ and its interaction with the oscillator(s) responsible for the tremor. ${ }^{11}$ Studies on the effect of wrist stretches on postural tremors have shown that larger amplitude tremors require larger wrist stretches to produce a similar degree of modulation. ${ }^{412}$ The same might be expected of electrical peripheral nerve stimulation (that is, larger amplitude tremors require larger stimuli), but the lack of correlation between tremor amplitude (measured at the wrist) and the resetting index found in this study does not support this hypothesis and cannot account for the variability in resetting indexes.

The behaviour of a tremor following peripheral nerve stimulation will also depend upon the interaction of the stimulus with the oscillators responsible for tremor. Further consideration of this factor is limited by our lack of knowledge about the location and nature of the oscillator(s) responsible for tremor. Several potential sites for tremor oscillators have been proposed, including peripheral reflex loops, ${ }^{13}$ dentato-rubro-olivary loops ${ }^{14}$ and thalamocortical loops, ${ }^{15}$ but there is no evidence that any one of these loops is wholly responsible for any individual tremor. It seems more likely that tremors depend on the interaction of many loops. ${ }^{11}$ This view of tremor, as an emergent characteristic of many interacting reflex loops, can be compared with a system of linked, nonlinear oscillators, ${ }^{11} 16$ the strength of the linkage between each oscillator reflecting the degree to which the different reflex loops interact with each other. Although the mathematical description of the behaviour of such a model is highly complex, ${ }^{11}$ the model may provide an explanation for the variability in susceptibility of tremors to peripheral nerve stimulation. The peripheral nerve shock might "reset" some oscillators in the system, partially influence others and not affect more remote oscillators. Subsequent behaviour of the tremor would then depend on the interaction of all oscillators in the system including those which had been "reset" and those which had been unaffected by the stimulus. Whether the tremor appeared "reset" would be decided by the relative balance of reset and unaffected oscillators (which in turn would depend on the size of the stimulus) as well as the strength of the linkage between oscillators.

\section{Conclusions}

Postural wrist tremors of essential tremor and Parkinson's disease as well as wrist tremors mimicked by normal subjects behave in similar ways to supramaximal stimulation of the median nerve at the elbow. The technique therefore cannot be used to differentiate the common causes of postural wrist tremors.

This work was supported in part by the Wellcome Trust.

We thank Mr R Bedlington for unfailingly maintaining and, where necessary, constructing the equipment used in this study.

1 Bathien N, Rondot P, Toma S. Inhibition and synchronisation of tremor induced by a muscle twitch. $\mathcal{f}$ Neurol Neurosurg Psychiatry 1980;43:713-8.

2 Findley LJ, Gresty MA, Halmagyi GM. Tremor, the cog wheel phenomenon and clonus in Parkinson's disease. f Neurol Neurosurg Psychiatry 1981;44:534-46.

3 Hömberg V, Hefter H, Reiners $\mathrm{K}$, Freund H-J. Differential effects of changes in mechanical limb properties on physiological and pathological tremor. 7 Neurol Neurosurg Psychiatry 1987;50:568-79.

4 Britton TC, Thompson PD, Day BL, Rothwell JC Findley LJ, Marsden CD. "Resetting" of postural tremors at the wrist with mechanical stretches in Parkinson's disease, essential tremor and normal subjects mimicking tremor. Ann Neurol 1992;31:507-14.

5 Lee RG, Stein RB. Resetting of tremor by mechanical Lee RG, Stein RB. Resetting of tremor by mechanical
perturbations: A comparison of essential tremor and perturbations: A comparison of essential tremor
parkinsonian tremor. Ann Neurol 1981;10:523-31.

6 Hufschmidt H-J. Proprioceptive origin of parkinsonian tremor. Nature 1963;200:367-8.

7 Mones RJ, Weiss AH. The response of tremor of patients with Parkinsonism to peripheral nerve stimulation. 7 Neurol Neurosurg Psychiatry 1969;32:512-8.

8 McLellan DL. Levodopa in parkinsonism: reduction in the electromyographic silent period and its relationship to tremor. F Neurol Neurosurg Psychiatry 1972;35:373-8.

9 Merton PA. The silent period in a muscle of the human hand. $\mathcal{F}$ Physiol 1951;114:183-98.

10 Marsden CD. Origins of normal and pathological tremor. In: Findley LJ, Capildeo R, eds. Movement disorders: tremor. London: Macmillan, 1984:37-84.

11 Rack PMH, Ross HF. The role of reflexes in the resting tremor of Parkinson's disease. Brain 1986;109:115-41.

12 Teräväinen H, Evarts E, Calne D. Effects of kinesthetic inputs on parkinsonian tremor. In Poirier LJ, Sourkes TL, Bedard PJ, eds. The extrapyramidal system and its disorders. Advances in neurology, vol 24 Raven Press: New York, 1979:161-73.

13 Lippold OCJ. Oscillation in the stretch reflex arc and the origin of rhythmical, 8-12c/s component of physiological tremor. $\mathcal{F}$ Physiol 1970;206:359-82.

14 Lamarre Y. Animal models of physiological, essential and parkinsonian-like tremors. In: Findley LJ, Capildeo R, eds. Movement disorders: tremor. London: Macmillan, 1984:183-94.

15 Lamarre Y, Joffroy AJ. Experimental tremor in monkey: activity of thalamic and precentral cortical neurones in the absence of peripheral feedback. In Poirier LJ, the absence of peripheral TL, Bedard PJ, eds. The extrapyramidal system Sourkes TL, Bedard PJ, eds. The extrapyramidal system and its disorders. Advances in neur

16 Elble RJ, Koller WC. Tremor. Baltimore: John Hopkins University Press, 1990. 